\title{
Estructura de tallas de tortuga pico de loro Lepidochelys olivacea (Testudines: Cheloniidae) en Tumbes, Perú
}

\author{
Size structure of Olive Ridley turtle Lepidochelys olivacea (Testudines: \\ Cheloniidae) in Tumbes, Peru
}

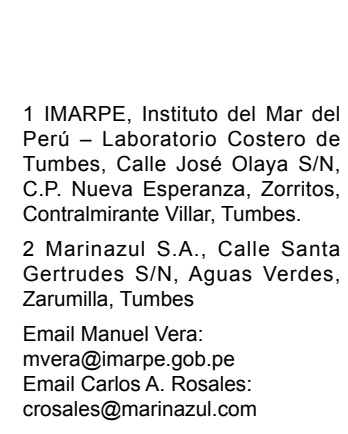

Presentado: $\quad$ 12/04/2012 Aceptado: $\quad 29 / 09 / 2012$ Publicado online: $10 / 11 / 2012$

\author{
Manuel Vera ${ }^{1}$ y Carlos A. Rosales ${ }^{2}$
}

\section{Resumen}

En el presente trabajo se analizó la estructura de tallas de ejemplares de tortuga pico de loro Lepidochelys olivacea, varados y capturados incidentalmente en las playas de Tumbes, Perú (338'9,5"S - 80³6'2,48”W y $3^{\circ} 57^{\prime} 21,3^{\prime \prime} \mathrm{S}-80^{\circ} 57^{\prime} 45,72^{\prime \prime} \mathrm{W}$ ), desde noviembre de 2006 a octubre de 2011. El área de estudio se dividió en tres zonas contiguas, limitadas por las quebradas más activas (Bocapán, El Rubio y Peña Negra). Se registraron 39 ejemplares ( $74,4 \%$ varados y $25,6 \%$ capturados incidentalmente), cuyas tallas variaron de 45 a $75 \mathrm{~cm}$ LCC $(62,5 \pm 5,7 \mathrm{~cm}$ LCC). El $64,1 \%$ correspondió a individuos sub-adultos $(<65 \mathrm{~cm} \mathrm{LCC} ; n=25)$. Espacialmente la mayor cantidad de registros ocurrieron en las zonas 3 y 2 , en las cuales porcentajes superiores al $60 \%$ se consideraron sub-adultos; mientras que en la zona 1, el $83 \%$ fue adulto. Temporalmente en la época lluviosa se registró la mayoría de las observaciones (59\%), en la cual el 78,3\% de ejemplares se consideró sub-adulto; siendo mayor que en la época seca $(43,8 \%)$. Las tallas presentaron diferencias significativas entre zonas y épocas climáticas. Se corroboró el patrón de distribución latitudinal por estados de madurez aparente (más adultos en el norte). La presencia del componente adulto indicaría que el litoral de Tumbes sería una potencial zona de anidamiento de L. olivacea, con mayores probabilidades de ovoposición en época seca, corroborada por los eventos de anidamiento recientes.

Palabras clave: Tortugas marinas; estados de madurez aparente; zona potencial de anidamiento; conservación; mar tropical.

\begin{abstract}
In this paper we analyzed the size structure of Olive Ridley turtle Lepidochelys olivacea, from individuals stranded and incidentally caught in beaches of Tumbes, Peru (3⒊ since November 2006 to October 2011. The study area was divided into three contiguous areas, which were limited by the most active streams (Bocapan, El Rubio and Peña Negra). There were 39 individuals (74.4\% stranded and $25.6 \%$ caught incidentally), with sizes ranged from 45 to $75 \mathrm{~cm} \mathrm{CCL}(62.5 \pm 5.7 \mathrm{~cm} \mathrm{CCL}) .64 .1 \%$ was considered sub-adult individuals $(<65 \mathrm{~cm} \mathrm{CCL}, \mathrm{n}=25)$. Spatially the largest number of records occurred in zones 3 and 2, in which percentages above $60 \%$ were considered sub-adults, while in zone 1, $83 \%$ was adult. Temporarily in the rainy season were registered the majority of the observations (59\%), in which $78.3 \%$ of specimens was sub-adult, being higher than in the dry season (43.8\%). Sizes showed significant differences between zones and climatic seasons. It was confirmed the latitudinal distribution pattern by apparent maturity stages (more adults in the north). Adults' occurrences suggest that Tumbes coast would be a potential nesting area of $L$. olivacea, most likely to oviposit in dry season, it which is supported by recent nesting events.
\end{abstract}

Keywords: Marine turtles; apparent maturity stages; conservation; potential nesting area; tropical sea.

\section{Introducción}

La tortuga golfina o tortuga pico de loro, Lepidochelys olivacea (Eschscholtz, 1829), es una de las cinco especies de tortugas marinas que usan el mar peruano como área de forrajeo en sus movimientos migratorios y como hábitat de desarrollo de individuos jóvenes (Hays-Brown \& Brown 1982, Rosales et al. 2010). Es categorizada por la Unión Internacional para la Conservación de la Naturaleza como especie vulnerable, pues su población está disminuyendo debido a la explotación selectiva (recolección de huevos y captura de adultos), captura incidental, deterioro de los lugares de anidamiento, enfermedades y depredación (AbreuGrobois \& Plotkin 2008).

Lepidochelys olivacea es más común en la zona norte del Perú, donde las temperaturas superficiales del mar son más elevadas (Hays-Brown \& Brown 1982), sin embargo su distribución también ha sido registrada en aguas costeras y oceánicas (IMAR-
PE 2011), Tumbes (Kelez et al. 2003, Forsberg 2008, Rosales et al. 2010), los puertos Salaverry, Chimbote, Cerro Azul e Ilo (Alfaro-Shigueto et al. 2002, 2007, 2008), la provincia de Pisco (Hays-Brown \& Brown 1982, de Paz et al. 2002, 2008, Manrique et al. 2003, 2004) y el puerto San Juan de Marcona (Majluf et al. 2002).

En Tumbes, L. olivacea es la segunda especie de tortuga marina con más registros de varamiento y capturas incidentales, después de Chelonia mydas, siendo más frecuente en Punta Picos, Canoas y Baja de Punta Mero (Rosales et al. 2010).

En este trabajo se analiza la estructura de tallas de los varamientos y capturas incidentales de L. olivacea en el litoral de Tumbes, Perú, con la finalidad de evaluar su condición poblacional, determinar el porcentaje de estados de madurez aparente (especialmente del componente adulto) y comprobar las diferencias en las tallas por zonas de registro y épocas climáticas. 


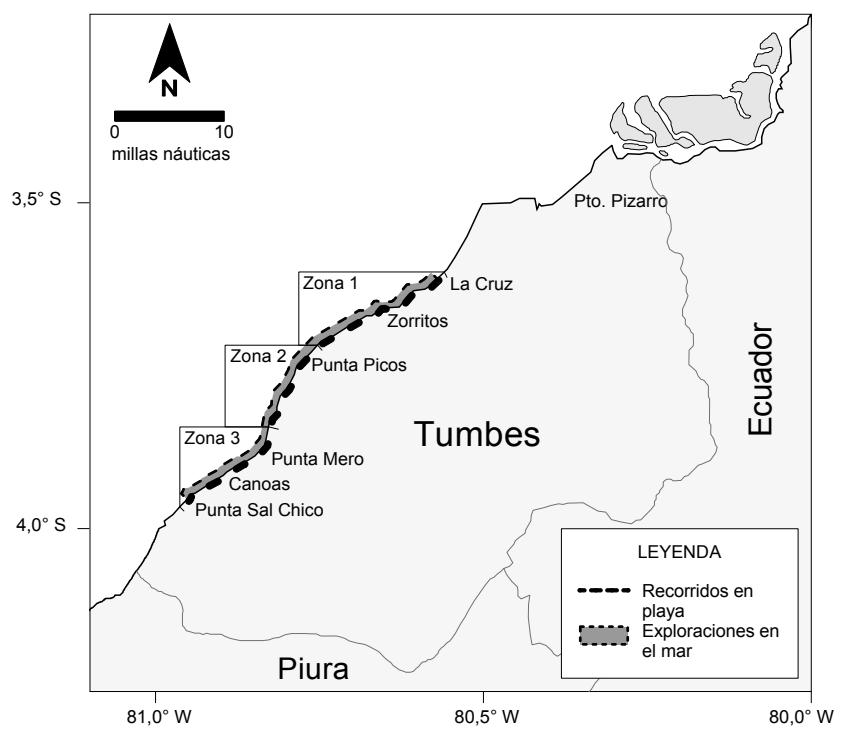

Figura 1. Área de estudio (línea y rectángulo punteados) de Lepidochelys olivacea en Tumbes, durante noviembre de 2006 a octubre de 2011.

\section{Material y métodos}

Área de estudio.- El estudio se realizó en el litoral de Tumbes, entre las playas Barrio El 19, La Cruz (3॰38'9,5"S - 80³6'2,48”W), y Punta Sal Chico (3०57'21,3”S $\left.80^{\circ} 57^{\prime} 45,72^{\prime \prime} \mathrm{W}\right)$, desde la línea de costa hasta una milla náutica de distancia (Fig. 1). El área fue dividida en tres zonas, cuyos límites fueron las quebradas más activas: 1 (Barrio El 19 a Quebrada Bocapán), 2 (Quebrada Bocapán a Quebrada El Rubio) y 3 (Quebrada El Rubio a Quebrada Peńa Negra). En esta área se distinguen dos épocas climáticas: una de lluvias de diciembre a mayo, y otra seca de junio a noviembre. Presenta algunas playas amplias que en su mayoría son de suave pendiente, con escasa vegetación y de fácil acceso desde el mar (Rosales et al. 2010), las que presentan numerosas quebradas que permanecen activas en épocas de lluvia y secas el resto del año (Ordinola et al. 2010). En el mar adyacente se ubican ocho bancos naturales de ostra Crassostrea iridescens, entre 2 y $7 \mathrm{~m}$ de profundidad; los principales bancos se ubican en Punta Sal Chico, Playa Florida y Bonanza; la pendiente de la plataforma continental es mayor en la zona sur (entre Lavejal y Punta Sal), predominando los sedimentos tipo arenoso y rocoso-arenoso (Ordinola et al. 2008).

Colecta de información y muestreo.- La información biométrica de $L$. olivacea provino de la base de datos de los registros de varamientos y capturas incidentales de tortugas marinas en Tumbes, recolectados en los recorridos de playas y exploraciones en el mar efectuados durante noviembre de 2006 a julio de 2008, y fue complementada con los datos recolectados en los recorridos de playas efectuados en agosto de 2009, octubre de 2010 y durante enero, febrero y octubre de 2011, por personal científico de IMARPE Tumbes.

Los ejemplares encontrados fueron identificados según Pritchard y Mortimer (2000) y Wyneken (2001), y medidos en su largo curvo del caparazón nucal-supracaudal (LCC), con una cinta métrica flexible (mm) (Bolten 2000).

Análisis de datos.- Se determinaron los parámetros biométricos de los ejemplares (mínimo, máximo, promedio y desviación estándar) y el porcentaje de los estados de madurez aparente (EMA: sub-adultos y adultos), considerando la talla promedio de hembras anidantes en la playa El Valle, Colombia $(65 \mathrm{~cm}$ LCC; Barrientos y Ramírez 2008).

Los valores de LCC se compararon entre zonas de registro (1, 2 y 3) y entre épocas climáticas (lluviosa y seca) con la prueba de Kruskal-Wallis, debido a que los datos no cumplieron los supuestos de normalidad y homogeneidad de varianzas.

\section{Resultados}

En Tumbes, durante noviembre de 2006 y octubre de 2011, se registraron 39 ejemplares de L. olivacea, correspondiendo el $74,4 \%$ a varamientos y el $25,6 \%$ a capturas incidentales. Los mayores registros ocurrieron en verano de 2007, primavera de 2010 y primavera de 2006 (Fig. 2) y principalmente en Punta Picos $(28,2 \%)$, en la zona 2 , y en Baja de Punta Mero $(25,6 \%)$ y Canoas (15,4\%), en la zona 3 (Tabla 1). Las tallas variaron de 45 a $75 \mathrm{~cm}$ LCC, con un promedio de 62,5 $\pm 5,7 \mathrm{~cm}$ LCC (Tabla 2).

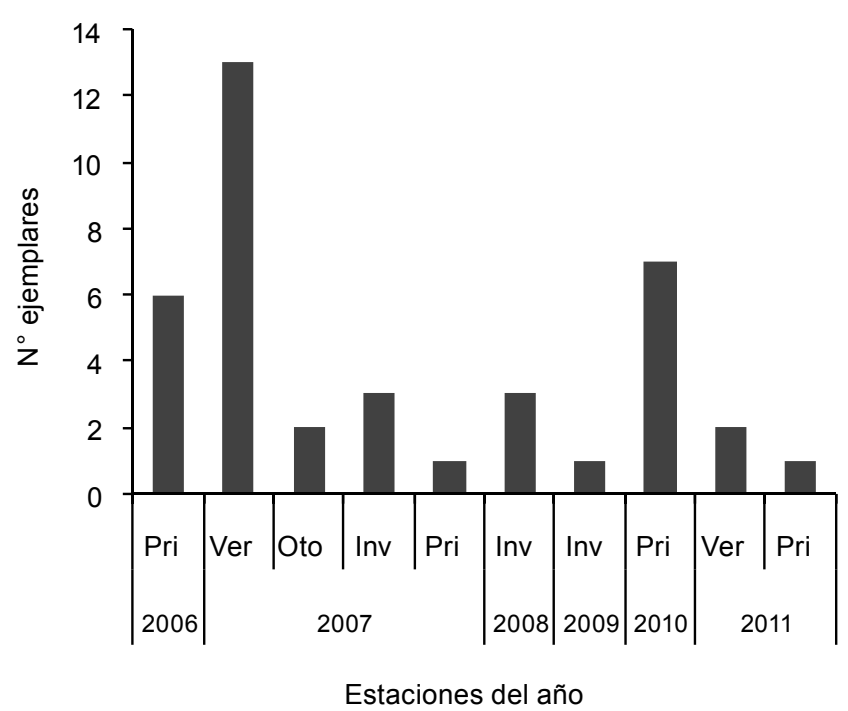

Figura 2. Número de ejemplares de Lepidochelys olivacea registrados por estaciones del año en Tumbes, durante noviembre de 2006 a octubre de 2011.
Tabla 1. Número de ejemplares de Lepidochelys olivacea por zonas de registro en Tumbes, durante noviembre de 2006 a octubre de 2011 .

\begin{tabular}{|c|c|c|c|c|}
\hline \multirow{2}{*}{ Zona } & \multirow{2}{*}{ Playa } & \multirow{2}{*}{ Localidad } & \multicolumn{2}{|c|}{ Total } \\
\hline & & & $\mathbf{n}$ & $\%$ \\
\hline \multirow{6}{*}{1} & Barrio El 19 & La Cruz & 1 & 16,7 \\
\hline & Quebrada Charán & La Cruz & 1 & 16,7 \\
\hline & Nueva Esperanza & Nueva Esperanza & 2 & 33,2 \\
\hline & Los Pinos & Zorritos & 1 & 16,7 \\
\hline & Tres Puntas & Tres Puntas & 1 & 16,7 \\
\hline & Total & & 6 & 100,0 \\
\hline \multirow{4}{*}{2} & Bonanza & Bonanza & 2 & 12,5 \\
\hline & Punta Picos & Acapulco & 11 & 68,7 \\
\hline & Lavejal & Lavejal & 3 & 18,8 \\
\hline & Total & & 16 & 100,0 \\
\hline \multirow{4}{*}{3} & Baja Punta Mero & Punta Mero & 10 & 58,8 \\
\hline & Canoas & Cancas & 6 & 35,3 \\
\hline & Punta Sal Chico & Punta Sal & 1 & 5,9 \\
\hline & Total & & 17 & 100,0 \\
\hline
\end{tabular}


Tabla 2. Estadísticos de tallas (largo curvo del caparazón nucal-supracaudal: LCC, cm) de Lepidochelys olivacea por estados de madurez aparente en Tumbes, durante noviembre de 2006 a octubre de 2011.

\begin{tabular}{lccc}
\hline \multirow{2}{*}{ Estadísticos } & \multicolumn{3}{c}{ Estados de madurez aparente } \\
\cline { 2 - 4 } & Sub-adulto & Adulto & Total \\
\hline $\mathrm{n}$ & 25 & 14 & 39 \\
Mínimo & 45 & 65 & 45 \\
Máximo & 64 & 75 & 75 \\
Promedio & 59,5 & 67,8 & 62,5 \\
DS & 4,5 & 2,9 & 5,7 \\
\hline
\end{tabular}

n: número de ejemplares, DS: desviación estándar.

De los ejemplares varados ( $\mathrm{n}=29$, todos muertos), el $48,3 \%$ fueron descuartizados para la comercialización de su carne, y en algunas ocasiones de su caparazón; y el restante (51,7\%) se mantuvo intacto, aunque el 53,3\% presentó lesiones en el cráneo, plastrón, extremidades y zona gular, que habrían sido causadas por ataques humanos o por colisión con embarcaciones pesqueras. El $37,9 \%$ se consideraron adultos $(67,3 \pm 2,3 \mathrm{~cm}$ LCC; $\mathrm{n}=11$ ).

En cuanto a los ejemplares capturados incidentalmente ( $\mathrm{n}=$ 10), el $90,0 \%$ fue sacrificado en playa para la comercialización de su carne, y en algunas ocasiones de su caparazón (de estos, el $44,4 \%$ fueron capturados vivos). Sólo un ejemplar fue hallado muerto (con signos de ahogamiento) y se mantuvo intacto. El $30,0 \%$ se consideraron adultos $(69,8 \pm 4,6 \mathrm{~cm} \mathrm{LCC}$; $=3)$.

Estructura de tallas según estados de madurez aparente.Del total de ejemplares, el 64,1\% se consideró sub-adulto; mientras que el 35,9\% adulto. Las tallas variaron en los sub-adultos de 45 a $64 \mathrm{~cm}$ LCC $(59,5 \pm 4,5 \mathrm{~cm}$ LCC; $\mathrm{n}=25)$, y en los adultos de 65 a $75 \mathrm{~cm}$ LCC $(67,8 \pm 2,9 \mathrm{~cm}$ LCC; $\mathrm{n}=14$ ) (Tabla 2).

Estructura de tallas según zonas de registro.- Lepidochelys olivacea fue registrada en toda el área de estudio, siendo más abundante en la zona 3, entre Quebrada El Rubio y Quebrada

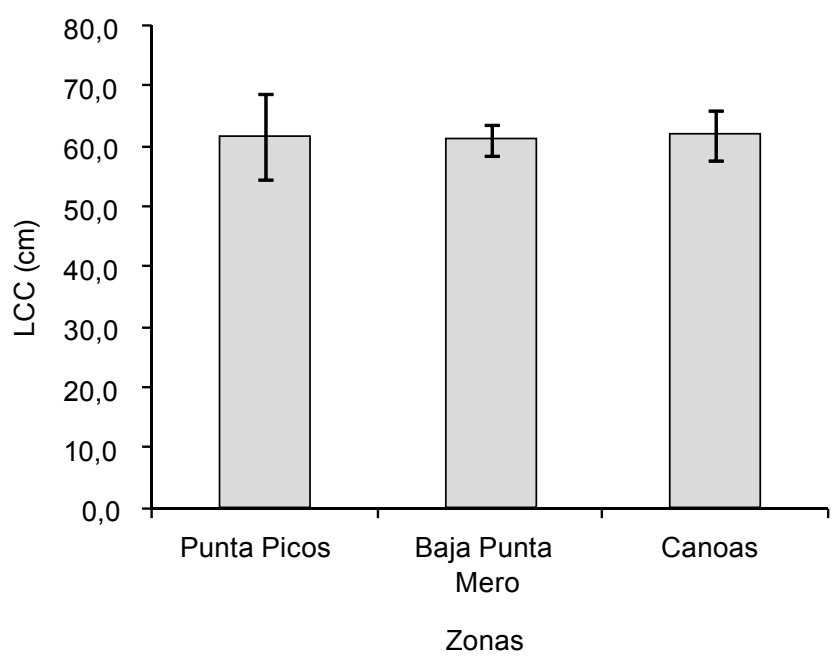

Figura 3. Promedio de largo curvo del caparazón nucal-supracaudal (LCC, cm), y desviación estándar, de Lepidochelys olivacea por zonas de registro en Tumbes, durante noviembre de 2006 a octubre de 2011.

Peńa Negra (Tabla 1). En la zona 1, las tallas variaron de 62 a $72 \mathrm{~cm}$ LCC $(67,5 \pm 3,3 \mathrm{~cm}$ LCC; $\mathrm{n}=6)$; mientras que en la zona 2, de 48 a $75 \mathrm{~cm}$ LCC $(62,8 \pm 6,1 \mathrm{~cm}$ LCC; $\mathrm{n}=16)$; y en la zona 3 , de 45 a $68 \mathrm{~cm} \mathrm{LCC}(60,5 \pm 5,0 \mathrm{~cm} \mathrm{LCC}$; $=17)$ (Tabla 3$)$.

En la zona 1, el 83,3\% de ejemplares se consideró adulto $(68,6 \pm 2,2 \mathrm{~cm}$ LCC; $\mathrm{n}=5)$; mientras que en las zonas 2 y 3 , el $62,5 \%(59,6 \pm 4,8 \mathrm{~cm}$ LCC; $\mathrm{n}=10)$ y $82,4 \%(59,4 \pm 4,6 \mathrm{~cm}$ LCC; $n=14$ ) se consideraron sub-adultos. Según la prueba de Kruskal-Wallis, las tallas presentaron diferencias significativas entre zonas de registro (Tabla 4).

Las playas con mayores registros (más de tres ejemplares) fueron Punta Picos (zona 2), Baja de Punta Mero y Canoas (zona 3), las que presentaron promedios de LCC similares durante el estudio (Fig. 3), con amplio rango de tallas (Tabla 3).

Estructura de tallas según épocas climáticas.- Del total de ejemplares, el 59\% se registró en la época lluviosa, y el restante

Tabla 3. Estadísticos de tallas (largo curvo del caparazón nucal-supracaudal: LCC, $\mathrm{cm}$ ) de Lepidochelys olivacea por zonas de registro en Tumbes, durante noviembre de 2006 a octubre de 2011.

\begin{tabular}{|c|c|c|c|c|c|c|c|}
\hline Zona & Playa & & $\mathbf{n}$ & Mín. & Máx. & Prom. & DS \\
\hline \multirow{5}{*}{1} & Barrio El 19 & & 1 & 69 & - & - & - \\
\hline & Quebrada Charán & & 1 & 72 & - & - & - \\
\hline & Nueva Esperanza & & 2 & 62 & 66 & 64,0 & 2,8 \\
\hline & Los Pinos & & 1 & 68 & - & - & - \\
\hline & Tres Puntas & & 1 & 68 & - & - & - \\
\hline \multirow{4}{*}{2} & & Total & 6 & 62 & 72 & 67,5 & 3,3 \\
\hline & Bonanza & & 2 & 64 & 69 & 66,5 & 3,5 \\
\hline & Punta Picos & & 11 & 48 & 75 & 61,7 & 7,0 \\
\hline & Lavejal & & 3 & 62 & 65 & 64,0 & 1,7 \\
\hline & & Total & 16 & 48 & 75 & 62,8 & 6,1 \\
\hline \multirow{4}{*}{3} & Baja Punta Mero & & 10 & 57 & 65 & 61,3 & 2,5 \\
\hline & Canoas & & 6 & 58 & 68 & 61,9 & 4,0 \\
\hline & Punta Sal Chico & & 1 & 45 & - & - & - \\
\hline & & Total & 17 & 45 & 68 & 60,5 & 5,0 \\
\hline
\end{tabular}

n: número de ejemplares; Mín.: LCC mínima; Máx.: LCC máxima; Prom.: promedio; DS: desviación estándar. 
Tabla 4. Prueba de Kruskal-Wallis para comparar el largo curvo del caparazón nucal-supracaudal (LCC, cm) de Lepidochelys olivacea entre zonas de registro y épocas climáticas, con el promedio y rango de tallas, en Tumbes, durante noviembre de 2006 a octubre de 2011.

\begin{tabular}{cccccc}
\hline Variable & Dimensión & $\mathbf{n}$ & Prom. \pm DS & Rango & Rango promedio \\
\hline \multirow{2}{*}{ Zona } & 1 & 6 & $67,5 \pm 3,3$ & $62-72$ & 31,50 \\
& 2 & 16 & $62,8 \pm 6,1$ & $48-75$ & 20,66 \\
& 3 & 17 & $60,5 \pm 5,0$ & $45-68$ & 15,32 \\
\hline \multirow{2}{*}{ Época climática } & Total & 39 & & & 16,09 \\
& Lluviosa & 23 & $60,5 \pm 5,6$ & $45-69$ & 25,63 \\
\hline
\end{tabular}

n: número de ejemplares; Prom.: promedio; DS: desviación estándar.

Tabla 5. Estadísticos de tallas (largo curvo del caparazón nucal-supracaudal: LCC, cm) de Lepidochelys olivacea por épocas climáticas y estados de madurez aparente (EMA) en Tumbes, durante noviembre de 2006 a octubre de 2011.

\begin{tabular}{|c|c|c|c|c|c|c|}
\hline Época & EMA & $\mathbf{n}$ & Mín. & Máx. & Prom. & DS \\
\hline \multirow{3}{*}{ Lluviosa } & Sub-adulto & 18 & 45 & 63,5 & 58,9 & 5,1 \\
\hline & Adulto & 5 & 65 & 68,5 & 66,5 & 1,7 \\
\hline & Total & 23 & 45 & 68,5 & 60,5 & 5,6 \\
\hline \multirow{3}{*}{ Seca } & Sub-adulto & 7 & 58 & 64 & 61,2 & 1,9 \\
\hline & Adulto & 9 & 65 & 75 & 68,6 & 3,3 \\
\hline & Total & 16 & 58 & 75 & 65,3 & 4,6 \\
\hline
\end{tabular}

n: número de ejemplares; Mín.: LCC mínima; Máx.: LCC máxima; Prom.: promedio; DS: desviación estándar.

en la época seca. En la época lluviosa, las tallas variaron de 45 a $69 \mathrm{~cm}$ LCC $(60,5 \pm 5,6 \mathrm{~cm} \mathrm{LCC} ; \mathrm{n}=23)$; mientras que en la época seca, de 58 a $75 \mathrm{~cm}$ LCC $(65,3 \pm 4,6 \mathrm{~cm} \mathrm{LCC}$; $=16)$ (Tabla 4). Los mayores promedios de LCC se registraron en la época seca, principalmente en 2009, 2010 y 2011 (Fig. 4).

En la época lluviosa, el 78,3\% de ejemplares se consideró sub-adulto $(58,9 \pm 5,1 \mathrm{~cm} \mathrm{LCC} ; \mathrm{n}=18)$; mientras que en la época seca, el $56,3 \%$ se consideró adulto $(68,6 \pm 3,3 \mathrm{~cm}$ LCC; $\mathrm{n}=9)$ (Tabla 5). Según la prueba de Kruskal-Wallis, las tallas presentaron diferencias significativas entre épocas climáticas (Tabla 4).

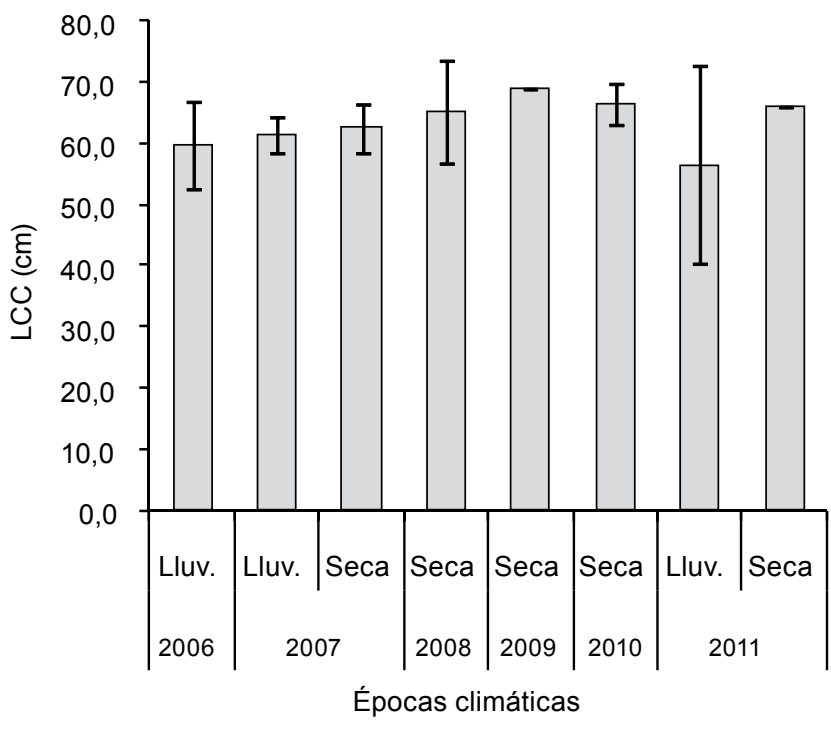

Figura 4. Promedio de largo curvo del caparazón nucal-supracaudal (LCC, cm), y desviación estándar, de Lepidochelys olivacea por épocas climáticas en Tumbes, durante noviembre de 2006 a octubre de 2011.

\section{Discusión}

Rosales et al. (2010), afirman que el litoral de Tumbes es una importante zona de forrajeo y desarrollo de L. olivacea, pues su presencia se registra casi todos los meses del año, y recomiendan realizar un trabajo más completo para evaluar si corresponde a un hábitat importante especialmente de ejemplares sub-adultos.

Nuestros resultados sobre la proporción de individuos adultos (35,9\%), sumado a los anteriores registros de hembras anidantes y de neonatos (Pérez et al. 2001, Kelez et al. 2009, Vera et al. 2010, Forsberg et al. 2012) señalan a Tumbes como la región más importante en Perú para L. olivacea.

El hallazgo de un mayor porcentaje de individuos sub-adultos en nuestras observaciones $(64,1 \%)$ indicaría una posible amenaza a la estabilidad de las poblaciones, porque son considerados estadios de vida extremadamente valiosos para la recuperación de las mismas (Crouse et al. 1987). Este porcentaje fue ligeramente inferior al registrado entre noviembre de 2006 y agosto de 2009 (75,9\%) por Rosales et al. (2010) para la misma área de estudio, y por De Paz et al. (2007), en Pisco en el verano de 2005 (72,7\%). Al respecto, la captura incidental de L. olivacea en el Perú llega a proporciones elevadas, como las observadas por De Paz et al. (2004) en el área de Pisco, entre julio de 1999 y junio de 2000, sobre el componente adulto (83\%); mientras en nuestra misma área de estudio, Rosales et al. (2010) afirman que los "balsilleros" capturaron sub-adultos en porcentajes superiores al 70\%, similar a lo registrado en nuestras observaciones.

Kelez et al. (2009), afirman que L. olivacea es una especie común en Perú y que posee un gran componente adulto en aguas costeras. $\mathrm{Al}$ respecto, el porcentaje de ejemplares adultos registrado por Rosales et al. (2010), para Tumbes (24,1\%), fue similar a los seńalados por De Paz et al. (2007), en Pisco (27,3\%), 
y por Kelez et al. (2009), en toda la costa peruana (33\%). En general, estos valores fueron ligeramente inferiores a lo registrado en nuestra investigación (35,9\%), lo cual sumado a su presencia tanto en épocas lluviosas como secas confirmaría que L. olivacea es una especie común en la costa peruana.

De los ejemplares registrados en la zona 1 de la región, el $83 \%$ se consideró adulto $(68,6 \pm 2,2 \mathrm{~cm} \mathrm{LCC} ; \mathrm{n}=5)$. Por el contrario, en las zonas 2 y 3 , porcentajes superiores al $60 \%$ se consideraron sub-adultos (59,6 $\pm 4,8 \mathrm{~cm}$ LCC; $\mathrm{n}=10$, y 59,4 44,6 cm LCC; $n=14$; respectivamente). Esto corroboraría el patrón de distribución latitudinal por estados de madurez aparente señalado por Rosales et al. (2010).

Los elevados porcentajes de ejemplares sub-adultos registrados en las zonas 2 y 3 (entre Bonanza y Punta Sal Chico), donde se ubican los principales bancos naturales de ostra Crassostrea iridescens (Ordinola et al. 2008), indicarían que esta zona es adecuada para la alimentación de la especie, debido a la mayor pendiente de la plataforma continental (especialmente entre Lavejal y Punta Sal) y al predominio de sedimentos tipo arenoso y rocoso-arenoso (Ordinola et al. 2008).

La talla promedio registrada en esta investigación $(62,5 \pm 5,7$ cm LCC; $\mathrm{n}=39$ ), similar a la señalada por Rosales et al. (2010) $(61,9 \pm 4,9$ cm LCC; $n=29)$, evidenció el patrón de distribución latitudinal referido por estos investigadores, debido a que fue ligeramente superior a la registrada en la zona sur del Perú por De Paz et al. (2004) (60,9 cm LCC).

A diferencia de lo señalado por Rosales et al. (2010), la comparación de LCC entre zonas de registro y épocas climáticas mostró diferencias significativas en las tallas. Al respecto, en la presente investigación, la delimitación de las zonas de registro (1, 2 y 3) abarcaron una distribución geográfica mayor, lo que pudo determinar las diferencias señaladas. En cuanto a las épocas climáticas, las diferencias encontradas pueden deberse a los mayores registros ocurridos en la época seca por el incremento de muestreos sistemáticos que habrían asegurado la recolección constante de muestras.

Las diferencias en tallas según épocas climáticas indicarían que habría mayores probabilidades de ovoposición en época seca, pues el 56,3\% de los ejemplares registrados en esta época se consideraron en estado adulto. Las fechas de anidamiento en Perú señaladas por Kelez et al. (2009), agosto de 2007, y Forsberg et al. (2012), octubre de 2011, confirman esta asunción.

La presencia de ejemplares adultos, especialmente en la zona 1 (entre Barrio El 19 y Quebrada Bocapán), indicaría que el litoral de Tumbes es una zona potencial de anidamiento de $L$. olivacea, condición sugerida por las actividades de anidamiento recientes de esta especie en Perú (Pérez et al. 2001, Kelez et al. 2009, Vera et al. 2010, Forsberg et al. 2012) y Ecuador (Alava et al. 2007). Al respecto, Kelez et al. (2009) mencionan que estas actividades pueden ser una re-colonización de las playas al límite sur de su distribución reproductiva en el Pacífico Oriental, lo que facilitaría la colonización de playas distantes de las playas natales respectivas. Sin embargo se recomienda que esta presunción sea comprobada realizando entrevistas a pobladores antiguos de la región.

Según Marcovaldi y Laurent (1996, en Martínez \& Páez 2000), los sitios de anidamiento de esta especie se caracterizan por ser playas amplias y de fácil acceso desde el mar, pudiendo realizarse en plataformas bordeadas por quebradas, donde la franja de arena es más amplia (Amorocho 1993, en Martínez \& Páez 2000), o cerca a la desembocadura de ríos y estuarios (Cornelius 1976, en Martínez \& Páez 2000). En Tumbes, las zonas de quebradas que discurren en épocas lluviosas y son observadas en muchos sectores del área de estudio (p.e. Quebrada Charán, Quebrada Bocapán, Quebrada El Rubio, Quebrada Peña Negra), pueden brindar condiciones adecuadas para el anidamiento de esta especie, pues poseen amplias franjas de arena.

Por lo tanto, de acuerdo a nuestras observaciones se puede afirmar que el litoral de Tumbes es una zona potencial de anidamiento de L. olivacea, con mayores probabilidades de ovoposición en época seca. En este sentido, y de acuerdo a las recomendaciones de Bjorndal (2000), se deben iniciar los estudios que evalúen los mecanismos regulatorios que controlan los parámetros demográficos, como los estudios de nutrición, hormonas, genética, fisiología, enfermedades y comportamiento en la regulación de la productividad de las poblaciones (crecimiento y reproducción) y sobre todo de mortalidad, para saber cuáles son sus fuentes y cuál es su nivel de impacto.

\section{Agradecimientos}

Al Dr. Jorge Llanos, por las facilidades brindadas para el registro de datos, a los Blgos. Elky Torres y Edward Morales, a los Ings. Elmer Ordinola, Solange Alemán y Johnny Robles, y al Téc. Manuel Vílchez, por su apoyo para el registro de datos, al Blgo. Percy Montero por su apoyo en la ubicación geográfica de las playas de muestreo, y a la Blga. Nelly de Paz, por su apoyo en la revisión, críticas y recomendaciones en la elaboración del presente trabajo.

\section{Literatura citada}

Abreu-Grobois A. \& P. Plotkin. 2008. (en línea). Lepidochelys olivacea. In: IUCN 2011. IUCN Red List of Threatened Species. Version 2011.2. <www.iucnredlist.org>. Acceso 17/11/2011

Alava J.J., P.C.H. Pritchard, J. Wyneken \& H. Valverde. 2007. First documented record of nesting by olive ridley turtle ( $L e$ pidochelys olivacea) in Ecuador. Chelonian Conservation \& Biology 6:282-285.

Alfaro-Shigueto J., M.F. van Bressem, D. Montes \& K. Onton. 2002. Turtle mortality in fisheries off the coast of Peru. In: A. Mosier, A. Foley and B. Brost, eds., Proceedings of the Twentieth Annual Symposium on Sea Turtle Biology and Conservation. NOAA Tech. Memo. NMFS-SEFSC-477. p. 91-93.

Alfaro-Shigueto J., P.H. Dutton, M.V. Bressem \& J.C. Mangel. 2007. Interactions between Leatherback turtles and Peruvian artisanal fisheries. Chelonian Conservation and Biology. 6(1):129-134.

Alfaro-Shigueto J., J.C. Mangel, P. Pajuelo, et al. 2008. Bycatch in Peruvian artisanal fisheries: gillnets versus longlines. In: A.F. Rees, M. Frick, A. Panagopoulou and K. Williams, eds., Proceedings of the Twenty-Seventh Annual Symposium on Sea Turtle Biology and Conservation. NOAA Technical Memorandum NMFS-SEFSC-569. p. 192.

Barrientos K. \& C. Ramírez. 2008. Estado actual de Lepidochelys olivacea en el Valle, Pacífico Chocoano, Colombia. In: S. Kelez, F. van Oordt, N. de Paz and K. Forsberg, eds. Libro de Resúmenes. II Simposio de Tortugas Marinas en el Pacifico Sur Oriental. p. 17-21. <http://www.ecOceanica. org/publicaciones $>$ Acceso 13/05/2010. 
Bjorndal K.A. 2000. Prioridades para la investigación en hábitats de alimentación. In: K.L. Eckert, K.A. Bjorndal, F.A. AbreuGrobois and M. Donnelly, eds. Técnicas de Investigación y Manejo para la Conservación de las Tortugas Marinas. 2000 (Traducción al español). IUCN/CSE Grupo Especialista en Tortugas Marinas Publicación No 4. p. 13-15.

Bolten A.B. 2000. Técnicas para la medición de tortugas marinas. In: K.L. Eckert, K.A. Bjorndal, F.A. Abreu-Grobois and M. Donnelly, eds. Técnicas de Investigación y Manejo para la Conservación de las Tortugas Marinas. 2000 (Traducción al español). IUCN/CSE Grupo Especialista en Tortugas Marinas Publicación No 4. p. 126-131.

Crouse D.T., L.B. Crowder \& H. Caswell. 1987. A stage-based population model for loggerhead sea turtles and implications for conservation. Ecology. 68(5):1412-1423.

De Paz N., J.C. Reyes \& M. Echegaray. 2002. Datos sobre captura, comercio y biología de tortugas marinas en el área de Pisco - Paracas. In: J. Mendo and M. Wolff, eds., I Jornada Científica "Bases ecológicas y socioeconómicas para el manejo de los recursos vivos de la Reserva Nacional de Paracas". Universidad Nacional Agraria La Molina. p. 125-129.

De Paz N., J.C. Reyes \& M. Echegaray. 2004. Capture and trade of marine turtles at San Andres, southern Peru. In: M.S. Coyne and R.D. Clark, eds. Procedings of the Twenty-First Annual Symposium on Sea Turtle Biology and Conservation. NOAA Technical Memorandum NMFS-SEFSC-528. p. 52-54.

De Paz N., J.C. Reyes, M. Echegaray, et al. 2007. Identificación y manejo de hábitats críticos de tortugas marinas en Perú: Paracas, estudio de caso. In: C. Guerra-Correa, A. Fallabrino, P. Bolados-Díaz and C. Turner, eds. VII Simposio Sobre Medio Ambiente. Estado Actual y Perspectivas de la Investigación y Conservación de las Tortugas Marinas en las Costas del Pacífico Sur Oriental. Antofagasta, Chile. p. 28. <http://www.uantof.cl/CREA/simpo\%20tortugas. pdf $>$ Acceso 13/05/2010.

De Paz N., J. Quiñones \& J. Zeballos. 2008. Análisis preliminar de la captura de las tortugas marinas en el Puerto de San Andrés - Pisco, Perú. In: S. Kelez, F. van Oordt, N. de Paz and K. Forsberg, eds., Libro de Resúmenes. II Simposio de Tortugas Marinas en el Pacífico Sur Oriental. p. 70.

Forsberg K. 2008. Proyecto tortugas marinas: iniciativas y esfuerzos para la conservación de las tortugas marinas en Tumbes. In: S. Kelez, F. van Oordt, N. de Paz and K. Forsberg, eds., Libro de Resúmenes. II Simposio de Tortugas Marinas en el Pacífico Sur Oriental. p. 69-70.

Forsberg K., A. Petit \& M. Arangüena. 2012. Avances en el estudio y monitoreo de anidación de tortugas marinas en el norte del Perú. In: A. Indacochea, ed. Libro de Resúmenes Digital. III Congreso de Ciencias del Mar del Perú. p. 72. $<$ https:// dl.dropbox.com/u/84039404/Aquahoy/LIBRO\%20 DE\%20RESUMENES\%20III\%20CONCIMAR\%202012. pdf $>$ Acceso 16/08/2012.

Hays-Brown C. \& W.M. Brown. 1982. Status of sea turtles in Southeastern Pacific: Emphasis on Peru. In: K.A. Bjorndal, ed. Biology and Conservation of Sea Turtles. Smithsonian Press, Washington D.C. p. 235-240.

IMARPE. 2011. Informe nacional sobre la conservación de las tortugas marinas en el Perú. Comisión Permanente del Pacífico Sur (CPPS). Plan de Acción para la Protección del Medio Marino y Áreas Costeras del Pacífico Sudeste. Lima, Perú. 72 pp.
Kelez S., X. Velez-Zuazo \& C. Manrique. 2003. Current status of sea turtles along the northern coast of Peru: preliminary results. In: J.A. Seminoff, ed., Proceedings of the TwentySecond Annual Symposium on Sea Turtle Biology and Conservation. NOAA Technical Memorandum NMFSSEFSC-503. p. 264-265.

Kelez S., X. Velez-Zuazo, F. Angulo \& C. Manrique. 2009. (en línea). Olive ridley Lepidochelys olivacea nesting in Peru: The southernmost records in the Eastern Pacific. Marine Turtle Newsletter 126:5-9. <http://www.seaturtle.org/mtn/ archives/mtn126/mtn126p5.shtml $>$ Acceso 05/04/2010.

Majluf P., E.A. Babcock, J.C. Riveros, et al. 2002. Catch and bycatch of sea birds and marine mammals in the small-scale fishery of Punta San Juan, Peru. Conservation Biology 16:1333-1343.

Manrique C., S. Kelez \& X. Velez-Zuazo. 2003. Hatchlings in Peru: the first headstarting experience, In: J.A. Seminoff, ed. Proceedings of the Twenty-Second Annual Symposium on Sea Turtle Biology and Conservation. NOAA Technical Memorandum NMFS-SEFSC-503. p. 99.

Manrique C., S. Kelez, X. Velez-Zuazo \& M. Williams de Castro. 2004. New morphometric data on captured sea turtles at San Andres Inlet, Pisco, Peru. In: M.S. Coyne and R.D. Clark, eds., Proceedings of the Twenty-First Annual Symposium on Sea Turtle Biology and Conservation. NOAA Technical Memorandum NMFS-SEFSC-528. p. 122-124.

Martínez L.M. \& V.P. Páez. 2000. Ecología de anidación de la tortuga golfina (Lepidochelys olivacea) en la Playa de La Cuevita, Costa Pacífica Chocoana, Colombia, en 1998. Actual Biol $22(73): 131-143$

Ordinola E., E. López, I. Gonzales, et al. 2010. Identificación y delimitación de bancos naturales de invertebrados marinos, zonas de pesca artesanal y áreas propuestas para maricultura en el litoral de la Región Tumbes. Inf. Interno Inst. Mar Perú. 83 pp. <http://www.imarpe.gob.pe/tumbes/ noticias/Ident_delimit_B_N Z 12-04-2010.pdf $>$ Acceso 09/12/2010.

Pérez O., A. Luján \& Z. Carrión. 2001. Crianza durante nueve meses en cautiverio de neonatos de Chelonia mydas "tortuga marina verde" en el Centro de Acuicultura La Tuna Carranza del FONDEPES - Tumbes. 9 pp.

Pritchard P.C.H. \& J.A. Mortimer. 2000. Taxonomía, morfología externa e identificación de las especies. In: K.L Eckert, K.A. Bjorndal, F.A. Abreu-Grobois and M. Donnelly, eds. Técnicas de Investigación y Manejo para la Conservación de las Tortugas Marinas. 2000 (Traducción al español). IUCN/CSE Grupo Especialista en Tortugas Marinas Publicación $\mathrm{N}^{\circ}$ 4. p. 23-41.

Rosales C.A., M. Vera \& J. Llanos. 2010. Varamientos y captura incidental de tortugas marinas en el litoral de Tumbes, Perú, Rev. peru. biol. 17(3): 293-301. <http://sisbib.unmsm. edu.pe/BVRevistas/biologia/v17n3/pdf/a04v17n3.pdf> Acceso 02/09/2011.

Vera M., J. Llanos, E. Torres, et al. 2010. Notas sobre neonatos de Lepidochelys olivacea (Testudines: Cheloniidae) en playa Nueva Esperanza, Tumbes, Perú. Inf. Inst. Mar Perú. 37(3-4): 161-166.

Wyneken J. 2001. The anatomy of sea turtles. US Department of Commerce, NOAA Tec. Memor. NMFS-SEFSC-470, $172 \mathrm{pp}$. 